\title{
Associations between the ICAM1 gene polymorphisms and atherosclerotic cardiovascular disease risk, including hypercholesterolemia, in rural Thais
}

\section{Naruemon Wechjakwen \\ Mahidol University \\ Amornrat Aroonnual \\ Mahidol University \\ Pattaneeya Prangthip \\ Mahidol University}

Ngamphol Soonthornworasiri

Mahidol University

Pornpimol Panprathip Phienluphon

Mahidol University

Jirayu Lainampetch

Mahidol University

Karunee Kwanbunjan ( $\nabla$ karunee.kwa@mahidol.ac.th )

Mahidol University

\section{Research Article}

Keywords: hypercholesterolemia, Intercellular adhesion molecule 1 gene, atherosclerotic cardiovascular disease, rural Thailand

Posted Date: March 4th, 2021

DOI: https://doi.org/10.21203/rs.3.rs-273467/v1

License: (9) This work is licensed under a Creative Commons Attribution 4.0 International License. Read Full License 


\section{Associations between the ICAM1 gene polymorphisms and atherosclerotic cardiovascular disease risk, including hypercholesterolemia, in rural Thais}

Naruemon Wechjakwen ${ }^{1}$, Amornrat Aroonnual ${ }^{1}$, Pattaneeya Prangthip ${ }^{1}$, Ngamphol Soonthornworasiri ${ }^{2}$, Pornpimol Panprathip Phienluphon ${ }^{1}$, Jirayu Lainampetch ${ }^{1}$, Karunee Kwanbunjan ${ }^{1, *}$

${ }^{1}$ Department of Tropical Nutrition and Food Science, Faculty of Tropical Medicine, Mahidol University, Bangkok, 10400, Thailand.

${ }^{2}$ Department of Tropical Hygiene, Faculty of Tropical Medicine, Mahidol University, Bangkok, 10400, Thailand.

*Corresponding author: Dr. Karunee Kwanbunjan

420/6 Ratchawithi Rd, Department of Tropical Nutrition and Food Science, Faculty of Tropical Medicine, Mahidol University, Bangkok, 10400, Thailand. Tel.: +66 886196555 , +66 81348 1909; fax: +66 26447934.

E-mail addresses:

NW: naruemon.wec@student.mahidol.ac.th

AA: amornrat.aro@mahidol.ac.th

PP: pattaneeya.pra@mahidol.ac.th

NS: ngamphol.soo@mahidol.ac.th

PPP: pornpimol.pan@mahidol.ac.th

JL: jirayu7208@gmail.com

KK: karunee.kwa@mahidol.ac.th 


\begin{abstract}
We determine the relationships between single nucleotide polymorphisms (SNPs), including rs5498 and rs287432 on Intercellular adhesion molecule 1 gene (ICAM1) and atherosclerotic cardiovascular disease (ASCVD) susceptibility in patients with hypercholesterolemia (HCL). The clinical characteristics of 278 participants were assessed, classified to group having HCL and without HCL. ICAM1 SNPs Genotyping was performed by DNA sequencing and ICAMI expression was measured using real-time PCR. Positive dominant model rs5498 subjects had twice the risk of HCL (P $=0.005)$. Participants with the rs5498 AG or GG variants and high ICAM1 mRNA expression ( $\geq 3.12)$ had 2.68 times the risk $(P=0.000)$, and those with a high LDL-C concentration $(\geq 3.36 \mathrm{mmol} / \mathrm{L})$ had 1.99 times the risk $(\mathrm{P}=0.014)$ of developing ASCVD compared with those with low ICAM1 mRNA and LDL-C levels. Interestingly, participants carrying the rs5498 AG or GG variants who had tachycardia (resting heart rates (RHRs) > 100 beats/min) had a 5.40times higher risk than those with a lower RHR $(\mathrm{P}=0.010)$. It may consider the $\mathrm{G}$ allele in ICAM1 rs5498 is associated with a higher risk of ASCVD in Thai people with HCL, and is also positively associated with ICAMI mRNA expression, LDL-C concentration, and RHR.
\end{abstract}

\title{
Abbreviations
}

ASCVD Atherosclerotic cardiovascular disease

HCL Hypercholesterolemia

SNPs Single nucleotide polymorphisms

ICAM1 Intercellular adhesion molecule 1

RHR Resting heart rate 


\section{Background}

Atherosclerotic cardiovascular disease (ASCVD), which involves the accumulation of plaque in the subendothelial space of arterial walls, is a fundamental process in the development of various cardiovascular diseases, including coronary heart disease (CHD), cerebrovascular disease (CeVD), and peripheral artery disease [1-2]. The many risk factors for ASCVD can be classified as modifiable risk factors, including dyslipidemia, high blood pressure, obesity, diabetes, alcohol consumption, and smoking, or non-modifiable risk factors, including age, sex, and genetic variation [2-7]. Hypercholesterolemia (HCL) is a major risk factor for ASCVD that is a component of an abnormal lipid metabolism [8-9] and promotes endothelial dysfunction, which is associated with ischemic heart disease and stroke [1012].

Intercellular adhesion molecule 1 gene $(I C A M 1$; CD54) is one of the immunoglobulin superfamily of cell adhesion molecules (CAMs) and is encoded by a gene located on chromosome 19p13.2 to 13.3. It plays a pivotal role in the firm attachment and transendothelial migration of leukocytes into the vascular intima [13-14]. Variants of the ICAMI gene have been shown to be important markers of endothelial dysfunction, which is the earliest stage in the pathogenesis of ASCVD [13, 15]. ICAMI expression and function depends on its complement of single nucleotide polymorphisms (SNPs) [16] and there are several known SNPs that are related to chronic inflammatory diseases, such as heart disease and stroke [17-19]. In Asian populations, rs5498 (A>G) and rs281432 (C>G) in the ICAM1 gene have been reported to affect the risks of ASCVD and other cardiovascular diseases (CVDs) [20-22].

Resting heart rate (RHR) is a non-invasive clinical parameter that is regulated by the autonomic nervous system (ANS). The effect of inflammatory mediators on endothelial cells inhibits the effects of the ANS. The consequence is excessive sympathetic tone, which has marked deleterious effects on the vascular system, including an increase in heart rate, the induction of arrhythmias, such as tachycardia (RHR > 100 beats/min) and atrial fibrillation (AF), and high blood pressure [23-26]. Furthermore, several previous studies have shown that a high heart rate affects several stages of the development of vascular diseases via endothelial function and genetic changes in several kinds of gene [27-32].

Although ICAMI gene variants have been reported to be important in patients with vascular diseases, there have been few studies of the relationships between ICAMI gene SNPs, RHR and HCL [18, 33, 34]. Therefore, we conducted a cross-sectional study to explore the relationships between the ICAM1 SNPs, rs5498 (A>G) and rs281432 (C>G), HCL, and ASCVD in Thai adults. We hypothesized that individuals with HCL who carry these genetic variants would show higher gene expression, impaired ANS function, and have a greater risk of ASCVD. If so, the identification of these polymorphisms would be useful for the prediction and prevention of ASCVD. 


\section{Methods}

\section{Study design and participants}

We conducted a cross-sectional study in which we randomly recruited 278 adults who had no evidence of systemic inflammation or infectious diseases in Sung Noen District, Nakhon Ratchasima Province, Thailand. The participants comprised 85 men and 193 women, aged between 35 and 60 years. The exclusion criteria were: 1) pregnancy or lactation; 2) presence of a serious health condition, including ischemic heart disease, stroke, peripheral artery disease, or any chronic disease; and 3) the regular use of medication. The participants were classified into two groups according to their lipid profile: 143 normal controls and 135 with HCL. The presence of HCL was diagnosed according to the National Cholesterol Education Program Adult Treatment Panel III (NCEP ATP III), as follows: serum total cholesterol $(\mathrm{TC}) \geq 5.17 \mathrm{mmol} / \mathrm{L}$, triglycerides $(\mathrm{TG}) \geq 1.70 \mathrm{mmol} / \mathrm{L}$, low-density lipoprotein-cholesterol $(\mathrm{LDL}-\mathrm{C}) \geq 3.37 \mathrm{mmol} / \mathrm{L}$, high-density lipoprotein-cholesterol $(\mathrm{HDL}-\mathrm{C}) \leq 1.04 \mathrm{mmol} / \mathrm{L}$ for men and $\leq 1.29 \mathrm{mmol} / \mathrm{L}$ in women, and TG/HDL-C ratio $\geq 1.75 \mathrm{mmol} / \mathrm{L}$. Each of these cut-off values is borderline high according to the reference ranges [35].

\section{Ethics declarations}

The study protocol was approved by the ethics committee of the Faculty of Tropical Medicine, Mahidol University (TMEC 18-026), and written informed consent was obtained from all the participants.

\section{Clinical and laboratory evaluation}

The participants underwent a physical examination to determine their health status. RHR and blood pressure were measured after $15 \mathrm{~min}$ of rest in the sitting position using an automatic oscillometer (Riester, Jungingen, Germany). Anthropometric measurements were made and classified according to the World Health Organization (WHO) AsiaPacific guidelines [36-37]. Body mass index (BMI) was calculated as body mass divided by height squared $\left(\mathrm{kg} / \mathrm{m}^{2}\right)$. Waist and hip circumference were measured using an inelastic tape-measure and waist-to-hip ratio (WHR) was calculated. These procedures were performed by the same trained nurses and researchers, using standardized techniques. Blood samples were collected from peripheral blood vessels after at least $8 \mathrm{~h}$ of fasting to determine lipid profile. Serum TC, TG, and HDL-C concentrations were determined using enzymatic colorimetric methods on a Cobas 6000 analyzer (Roche Diagnostics International Ltd., Basel, Switzerland). Serum low-density lipoprotein-cholesterol (LDL-C) concentration was calculated using the Friedewald equation in participants with a TG concentration $<4.5$ $\mathrm{mmol} / \mathrm{L}$ as follows: $[\mathrm{LDL}-\mathrm{C}]=[\mathrm{TC}]-([\mathrm{TG}] / 2.2)-[\mathrm{HDL}-\mathrm{C}][38]$ 


\section{Genomic DNA extraction, genotyping of the ICAM1 rs5498 (AG) and rs281432 (CG) polymorphisms,} and DNA sequencing

Genomic DNA was extracted from peripheral blood leukocytes using a FlexiGene DNA kit (Qiagen, Hilden, Germany), according to the manufacturer's instructions. The ICAM1 rs5498 and rs281432 polymorphisms were identified by PCR using KAPA2G Fast HotStart master mix (Kapa Biosystems, Wilmington, MA, US) containing $1 \mu \mathrm{L}$ of template DNA and $0.5 \mu \mathrm{L}$ of each primer (forward and reverse). The final volume was adjusted to $25 \mu \mathrm{L}$ with nuclease-free water. The PCR amplification conditions comprised an initial denaturation step of $5 \mathrm{~min}$ at $95^{\circ} \mathrm{C}$, followed by 35 cycles of denaturation for $30 \mathrm{~s}$ at $95^{\circ} \mathrm{C}$, annealing for $45 \mathrm{~s}$ at $60^{\circ} \mathrm{C}$, and extension for $30 \mathrm{~s}$ at $72^{\circ} \mathrm{C}$, with a final extension of $5 \mathrm{~min}$ at $72^{\circ} \mathrm{C}$. The primers used for ICAM1 rs5498 were (forward) $5^{\prime}$-TTG AGG GCA CCT ACC TCT GT-3' and (reverse) 5'-CAT TAT GAC TGC GGC TGC TA-3', yielding an amplicon of size $214 \mathrm{bp}$, and those for rs281432 were (forward) 5'-GAG GAG CTG GGA CTT TCC TT-3' and (reverse) 5'-CCC TGA CCT GCA GTC CTT TA-3', yielding an amplicon of $231 \mathrm{bp}$. The amplified DNA was evaluated by agarose gel electrophoresis. The ICAMI rs5498 and rs281432 regions were also directly genotyped by DNA sequencing by Bio Basic Asia Pacific Pte. Ltd. (Bukit Batok, Singapore).

\section{RNA extraction and real-time PCR analysis}

To measure ICAMI mRNA expression in peripheral blood mononuclear cells, RNA was isolated using NucleoZol reagent (Macherey-Nagel, Duren, Germany), according to the manufacturer's instructions. To synthesize cDNA for real-time PCR, $2 \mu \mathrm{g}$ RNA was reverse transcribed and genomic DNA was removed using a ReverTra Ace ${ }^{\mathrm{q}} \mathrm{qPCR}$ RT Master Mix and gDNA remover from Toyobo Co. Ltd. (Osaka, Japan). Real-time PCR was performed according to the manufacturer's instructions using iTaq ${ }^{\mathrm{TM}}$ Universal SYBR ${ }^{\circledR}$ Green Supermix (Bio-Rad Laboratories Inc., Hercules, CA, US). The primer sequences were as follows: (forward) 5'-GCA TCC TGG GCT ACA CTG AG-3' and (reverse) 5'TGC TGT AGC CAA ATT CGT TG-3' for the reference gene GAPDH, and (forward) 5'-ACA GTC ACC TAT GGC AAC GAC-3' and (reverse) 5'-GTC ACT GTC TGC AGT GTC TCC T-3' for ICAM1. The relative ICAM1 mRNA expression was normalized to that of GAPDH. All amplification reactions were performed in duplicate and the results were quantified using the $2^{-\Delta \Delta \mathrm{Ct}}$ method.

\section{Statistical analysis}

Statistical analyses were conducted using SPSS 18.0 (IBM, Inc., Armonk, NY US). The data were first tested for normal distribution using the Kolmogorov-Smirnov test. Differences between groups were identified using Student's $t$ test or ANOVA if the data were normally distributed, and the Mann-Whitney $U$ test or Kruskal-Wallis test for non- 
normally distributed data. Twin sets of categorical data were compared using the chi-square test. The genotype frequencies of the ICAMI variants were analyzed for Hardy-Weinberg equilibrium (HWE) in the HCL and control groups. To determine the relationships between ICAM1 SNPs and HCL, odds ratios (OR) and 95\% confidence intervals (95\% CIs) were calculated using logistic regression. Comparisons of the distributions of the alleles and genotypes were performed using the chi-square test. Statistical significance was set at $P<0.05$.

\section{Results}

\section{Clinical and laboratory characteristics of the participants with HCL and normal controls}

Table 1 shows the clinical and laboratory characteristics of participants with HCL and normal controls. The prevalence of HCL was $49 \%$ (135/278). The sex and age ratios in the groups were identical. The SBP, DBP, and RHR of the participants were significantly higher than those of normal controls. Likewise, the body composition of the groups differed: BMI, WC, HC, and WHR were significantly higher in the HCL group than the normal control group. As expected, the serum TC, LDL-C, and TG concentrations and the TG/HDL-C ratio of the HCL group were significantly higher than those of normal controls (all $\mathrm{P}=0.000$ ). Moreover, there were no significant differences in behavioral characteristics, including in the prevalences of cigarette smoking, alcohol consumption, and exercise, between the HCL and normal control groups. However, there were no differences in HDL-C concentration or ICAMI mRNA expression between the two groups $(\mathrm{P}=0.218$ and $\mathrm{P}=0.096$, respectively $)$.

\section{ICAM1 rs5498 and rs281432 genotype}

The rs5498 and rs281432 polymorphisms of ICAMI were genotyped. As shown in Fig. 1 the heterozygous and homozygous variants of ICAM1 rs5498 were identified as AG and GG, while AA was the wild-type genotype, and those of rs281432 were CG and GG, and CC, respectively.

\section{Associations of the ICAM1 rs5498 and rs281432 polymorphisms with HCL}

The distributions of the genotypes were consistent with the presence of Hardy-Weinberg equilibrium in both groups. As shown in Table 2, the genotype distribution of ICAM1 rs5498 (A > G) in the participants with HCL was GG (10\%), AG (43\%), and AA (47\%), which was significantly different to that of the control participants, who had a distribution of GG (7.0\%), AG (29\%), and AA (64\%). Allele frequency analysis for ICAM1 rs5498 showed that the G allele was more frequent in the participants with HCL than in normal controls (OR 1.69; 95\% CI 1.15-2.47). Moreover, the genetic model of ICAM1 rs5498 was evaluated, which demonstrated a statistically significant association between ICAMI rs5498 and HCL in the dominant model (AA vs. AG+GG) (OR 2.00, 95\% CI 1.24-3.23). This also showed a positive association between the presence of both HCL and ICAM1 rs5498 and a high risk of ASCVD. However, there was no 
significant difference in the genotype distribution of ICAM1 rs281432 (C > G) between the HCL and normal groups (P $=0.972$ ). The risk factors in genetic models of rs281432 (additive, dominant, and recessive) were compared by logistic regression analysis and no significant associations were found using these genetic models.

\section{Comparison of the clinical and laboratory characteristics of participants with rs5498 and rs281432 of ICAM1 polymorphisms using the dominant model}

We found strong associations between the SNPs and HCL using the dominant model (Table 2). Therefore, we further analyzed the relationships between ICAMI SNPs, HCL, and other variables. The mean values of clinical and laboratory characteristics were calculated according to allele distribution (Table 3). Participants carrying the G allele (AG and GG genotype) of rs5498 had a higher RHR than those carrying only the A allele (AA vs. AG + GG: $77.26 \pm 11.44$ bpm vs. $81.58 \pm 12.19 \mathrm{bpm} ; \mathrm{P}=0.003)$. Furthermore, participants carrying the $\mathrm{G}$ allele of rs5498 had higher serum TC and LDL-C concentrations and ICAMI mRNA expression than those carrying only the A allele $(\mathrm{P}=0.004,0.002$, and $<$ 0.001, respectively). Moreover, the prevalence of smoking was higher in rs5498 wild-type (AA) participants than in those with the variants $(\mathrm{AG}+\mathrm{GG})(\mathrm{P}=0.035)$. However, there were no differences between these groups with respect to alcohol consumption, exercise, serum TG concentration, or TG/HDL ratio. Furthermore, there were no significant relationships between the ICAM1 rs281432 genotype and clinical and laboratory characteristics.

\section{Relationships between ICAM1 rs5498 polymorphism, HCL, and other ASCVD risk factors}

According to the NCEP ATP III and previous studies, there are multiple ASCVD risk factors related to HCL, including overweight/obesity, dyslipidemia, high ICAMI mRNA expression, and ICAMI SNPs. Therefore, we assessed the ASCVD risk factors according to the genetic dominant model in participants with HCL with the G allele (variants, AG+GG) and in those without the G allele (wild-type, AA) (Fig. 2). We found that the mean RHR of participants with HCL and the AG or GG genotype was significantly higher than that of normal controls with the AA genotype (P < 0.001). In addition, the BMI of participants with HCL and normal controls with the AG or GG genotype was higher than that of participants with HCL and normal controls with the AA genotype $(\mathrm{P}=0.042$ for trend). There were also significant differences in lipid profile: participants with the AG or GG genotypes had significantly higher TC, LDL-C, and TG/HDL ratio than in normal controls with any of the genotypes $(\mathrm{P}<0.001)$. The TG concentration in participants with AG or GG was higher than in normal controls with any genotype or in participants with HCL and the AA genotype $(\mathrm{P}<0.001)$. Furthermore, we found higher expression of ICAMI mRNA in participants with HCL and normal controls with AG or GG genotypes than in either group with an AA genotype $(\mathrm{P}<0.05)$. However, there were no differences in HDL-C concentrations among the groups. These results imply that individuals carrying the $\mathrm{G}$ allele of 
rs5498, whether hypercholesterolemic or normocholesterolemic, have a higher risk of ASCVD. In contrast, participants carrying the rs281432 variants did not show this association.

\section{The effect of the interaction of the ICAM1 rs5498 polymorphism and ASCVD risk factors}

The combined impacts of ICAMI genotype and specific ASCVD risk factors were determined. In the participants, values above the 75th percentile for RHR ( $\geq 86$ beats $/ \mathrm{min}$ ) or tachycardia ( $>100$ beats $/ \mathrm{min})$, abnormal lipid profile (borderline high values for each parameter), and values above the 75th percentile for ICAM1 mRNA expression ( $\geq 3.12$ arbitrary units) were defined as confering a high risk of ASCVD. Participants with the rs5498 AG+GG genotype and a high serum LDL-C concentration ( $\geq 3.36 \mathrm{mmol} / \mathrm{L}$ ) were at higher risk of ASCVD (OR 2.08; 95\% CI 1.19-3.65) than those with a low serum LDL-C concentration. There was also a 2.47-fold higher ASCVD risk (95\% CI 1.54-4.66) in participants with an $I C A M 1$ mRNA expression level of $\geq 3.12$ than in those with an ICAMI mRNA expression level of <3.12. Furthermore, we found a robust interaction between the ICAM1 rs5498 polymorphism and high RHR. The presence of the rs5498 variant (AG+GG) and RHR above the $75^{\text {th }}$ percentile or tachycardia increased the risk of ASCVD (OR 1.60; 95\% CI 0.92-2.76 and OR 4.92; 95\% CI 1.33-18.23, respectively) over that associated with a lower RHR. This finding suggests that the higher the RHR, the higher the risk of developing ASCVD. All the ORs quoted were adjusted for sex, age, smoking status, alcohol consumption status, and exercise habits (Table 4). However, we did not identify any significant interactions between the ICAM1 rs281432 polymorphism and ASCVD risk factors.

\section{Discussion}

In the present cross-sectional study we investigated the relationships between the ICAM1 rs5498 polymorphism and modifiable and non-modifiable risk factors for ASCVD. First, we found a significant association between the rs5498 dominant model and HCL in a sample of adult Thai people. Second, rs5498 variants were associated with multiple risk factors for ASCVD: RHR, TC, LDL-C, and ICAM1 mRNA expression. Third, participants with HCL and an rs5498 variant had higher RHR, BMI, TC, LDL-C, TG, TG/HDL-C ratio, and ICAMI mRNA expression. Finally, participants with an rs5498 variant had a higher RHR, higher serum LDL-C concentration, and higher expression of ICAMI mRNA than those with the wild-type AA genotype. These results demonstrate that in Thai adults variation at the ICAMI rs5498 locus is associated with HCL and high RHR, which are risk factors for ASCVD, and this is consistent with the findings of previous studies conducted in other populations [24, 26, 39, 40].

The pathogenesis of ASCVD is considered to be multifactorial, involving oxidative stress and an upregulation of CAMs expression, induced by proinflammatory cytokines, including interleukin (IL)-6, tumor necrosis factor (TNF)- $\alpha$, and IL-1 $\beta$, all of which are consequences of overweight/obesity, HCL, and related risk factors, including smoking and 
alcohol consumption $[5,34]$. The accumulation of lipids, especially LDL-C, is thought to initiate oxidative stress, and oxidized LDL and macrophage recruitment amplify the inflammation, resulting in endothelial dysfunction in arterial walls $[1,41]$. High expression of ICAMI is a result of the chronic inflammation of the arteries and leads to the migration of leukocytes into the intima [13]. The common polymorphism at rs5498 in exon 6 of the ICAM1 gene results in the substitution of glutamate for lysine (K469E) in the immunoglobulin-like domain 5 of the ICAM-1 protein, which is the result of an A-to-G substitution [42]. This polymorphism has also been suggested to affect mRNA splicing patterns, with effects on cell-cell interactions and the inflammatory response (Fig. 3) [39, 40]. These changes have been shown to be involved in the etiology of ASCVD [43].

A previous study by Sarecka-Hujar et al. showed that individuals possessing the AG or GG genotypes at ICAMI rs5498 tended to have higher TC and LDL-C concentrations than those possessing the AA genotype, and were therefore at higher risk of developing coronary artery disease [39]. This is consistent with the present finding of significant positive relationships between the rs5498 variant and high serum TC and LDL-C concentrations. In addition, we identified associations with other risk factors (BMI, TG, and TG/HDL-C ratio), all of which were high in participants with HCL who were carrying the rs5498 variant. Therefore, we conclude that the rs5498 variant predisposes toward HCL, which may progress to ASCVD.

As mentioned above, high expression of ICAMI is a vascular biomarker of endothelial dysfunction. The genetic variants of ICAMI affect its expression and promote the development of ASCVD, as shown by numerous studies. In addition, Iwao et al. found that these variants affect RNA splicing: cells with the GG genotype express less ICAM-1-S mRNA than those with an AA genotype, and the authors suggested that the 548G>A (E469K) polymorphism modifies inflammatory responses by altering cell-cell interactions and regulating apoptosis [40]. Similarly, in the present study, we found a significant association between the rs5498 polymorphism and high expression of ICAM1 mRNA. Therefore, a combination of HCL with the rs5498 variants may promote the development of ASCVD by increasing ICAM1 expression.

The autonomic nervous system regulates homeostasis and consists of two major branches: the sympathetic and the parasympathetic nervous systems. Autonomic imbalance can result from oxidative stress, systemic inflammation, endothelial dysfunction, and genetic variation, with HCL having an additive effect to these defects [27, 44]. The imbalance in the autonomic nervous system is characterized by hyperactivity of the sympathetic system and hypoactivity of the parasympathetic system, which results in high RHR and blood pressure and vascular dysfunction (Fig. 3). High RHR is considered to be a risk factor for heart disease, and can reflect several types of arrhythmia, such as supraventricular tachycardia, atrial fibrillation, sinus tachycardia, and ventricular tachycardia $[26,45,46]$. 
Thayer et al. suggested that such autonomic imbalance might be the final common pathway linking health conditions, including CVD. Therefore, a change in lifestyle that ameliorates biological risk factors and reduces this autonomic imbalance may reduce the risk of vascular disease [23, 47-50]. Böhm et al. also suggested that heart rate is associated with cardiovascular outcomes and other conditions, including endothelial dysfunction [26]. In addition, Nanchen et al. reported an association between RHR and incident heart failure in a population-based cohort study of healthy adults without pre-existing overt heart disease. They suggested that the risk of heart failure increases in men with each increment of 10 beats per minute in RHR [24]. Likewise, we have shown an association between other ASCVD risk factors and a high RHR: the rs5498 variant was associated with a 1.74-fold higher risk of ASCVD than wild-type when RHR was $\geq 86$ beats/min. Furthermore, the ICAM1 rs5498 variant was associated with a 5.40-fold higher risk in the presence of tachycardia ( $\geq 100$ beats/min). This is consistent with systemic inflammation, oxidative stress, and endothelial dysfunction worsening in individuals with HCL and the ICAMI variant as heart rate rises. However, we did not identify an association between rs281432 (C > G) and HCL. In contrast, and similar to the present findings, Yang et al. found no significant difference in the prevalences of the rs281432 genotypes in patients with or without coronary atherosclerosis [22].

The strengths of the present study were that we studied the effect of key genetic variants in a population with the same race, age group, lifestyle, and environment. The sequencing technique we used was able to precisely identify the SNPs. Moreover, we were able to confirm the association between ICAMI expression and endothelial dysfunction in ASCVD. However, the cross-sectional design was a limitation, such that we could not show a cause-and-effect relationship between the ICAMI rs5498 polymorphism, HCL, and ASCVD.

\section{Conclusions}

we have shown relationships between the ICAM1 rs5498 polymorphism and HCL and the related ASCVD risk factors in Thai adults. The underlying mechanisms for this association will involve interactions between abnormal lipid profile, oxidative stress, inflammatory infiltration, and circulating cell adhesion molecules. Although previous studies have indicated that the identification of the ICAMI gene variant might be predictive of atherosclerosis in CVD patients, here we show that the variant may predict HCL and therefore may be used in the prevention of ASCVD. 


\section{References}

1. Linton, M. F. et al. The role of lipids and lipoproteins in atherosclerosis. In Endotext (eds. Feingold, K. R. et al.) 1-96 (MDText.com, Inc., South Dartmouth (MA), 2000).

2. Mach, F. et al. 2019 ESC/EAS guidelines for the management of dyslipidaemias: lipid modification to reduce cardiovascular risk: the task force for the management of dyslipidaemias of the European Society of Cardiology (ESC) and European Atherosclerosis Society (EAS). Eur. Heart J. 41, 111-188, https://doi.org/10.1093/eurheartj/ehz455 (2020).

3. Arnett, D. K. et al. 2019 ACC/AHA guideline on the primary prevention of cardiovascular disease: a report of the American College of Cardiology/American Heart Association: task force on clinical practice guidelines. J. Am. Coll. Cardiol. 74, e177-e232, https://doi.org/10.1016/j.jacc.2019.03.010 (2019).

4. Virani, S. S. et al. Heart disease and stroke statistics-2020 update: a report from the American Heart Association. Circulation 141, e139-e596, https://doi.org/10.1161/CIR.0000000000000757 (2020).

5. $\quad$ Boehme, A.K., Esenwa, C. \& Elkind, M. S.V. Stroke risk factors, genetics, and prevention. Circ. Res. 120, 472495, https://doi.org/10.1161/CIRCRESAHA.116.308398 (2017).

6. $\quad$ van Dijk, S. J., Tellam, R. L., Morrison, J. L., Muhlhausler, B.S. \& Molloy, P. L. Recent developments on the role of epigenetics in obesity and metabolic disease. Clin. Epigenetics 7, 66, https://doi.org/10.1186/s13148-015-01015 (2015).

7. Chen, J. Y., Chang, K. C. \& Liou, Y. M. Matrix metalloproteinase 11G/2G gene polymorphism is associated with acquired atrioventricular block via linking a higher serum protein level. Sci. Rep. 10, 9900, https://doi.org/10.1038/s41598-020-66896-9 (2020).

8. Verbeek, R. et al. Cardiovascular disease risk associated with elevated lipoprotein(a) attenuates at low lowdensity lipoprotein cholesterol levels in a primary prevention setting. Eur. Heart J. 39, 2589-2596. https://doi.org/10.1093/eurheartj/ehy334 (2018).

9. Martinez-Hervas, S. \& Ascaso, J. F. Hypercholesterolemia. In Encyclopedia of Endocrine Diseases 2nd edn (eds Huhtaniemi, I. \& Martini, L.) 320-326 (Academic Press, Oxford, 2019).

10. Manktelow, B. N. \& Potter, J. F. Interventions in the management of serum lipids for preventing stroke recurrence. Cochrane Database Syst Rev, 1-22, https://doi.org/10.1002/14651858.CD002091.pub2 (2009).

11. O’Gara, P. T. et al. 2013 ACCF/AHA guideline for the management of ST-elevation myocardial infarction. Circulation 127, e362-e425, https://doi.org/10.1161/CIR.0b013e3182742cf6 (2013). 
12. Grundy, S. M. et al. Implications of recent clinical trials for the National Cholesterol Education Program Adult Treatment Panel III guidelines. Circulation 110, 227-239, https://doi.org/10.1161/01.CIR.0000133317.49796.0E (2004).

13. Haught, W. H. et al. Alterations in circulating intercellular adhesion molecule-1 and L-selectin: further evidence for chronic inflammation in ischemic heart disease. Am. Heart J. 132, 1-8, https://doi.org/10.1016/S00028703(96)90383-X (1996).

14. Di Pietro, N., Formoso, G. \& Pandolfi, A. Physiology and pathophysiology of oxLDL uptake by vascular wall cells in atherosclerosis. Vascul. Pharmacol. 84, 1-7, https://doi.org/10.1016/j.vph.2016.05.013 (2016).

15. Smiljić, S., Mijović, M. \& Savić, S. Biomarkers of endothelial dysfunction in cardiovascular diseases. Med. Pregl. 70, 53-57, https://doi.org/10.2298/MPNS1702053S (2017).

16. Shaker, O. et al. Role of ICAM-1 and E-selectin gene polymorphisms in pathogenesis of PAOD in Egyptian patients. Vasc. Health Risk Manag. 6, 9-15, PMC: 2828103 (2010).

17. Gazi, E. et al. Intercellular adhesion molecule-1 K469E and angiotensinogen T207M polymorphisms in coronary slow flow. Med Princ Pract 23, 346-350, https://doi.org/10.1159/000363451 (2014).

18. Li, D., Qu, C. \& Dong, P. The ICAM-1 K469E polymorphism is associated with the risk of coronary artery disease: a meta-analysis. Coron. Artery Dis. 25, 665-670, https://doi.org/10.1097/MCA.0000000000000136 (2014).

19. Wang, D. et al. Association of polymorphism in ICAM-1 (K469E) and cytology parameters in patients' initial blood test with acute ischemic stroke. Genet. Mol. Res. 14, 15520-15529, https://doi.org/10.4238/2015.December.1.2 (2015).

20. Chou, C. H. et al. Impact of intercellular adhesion molecule-1 genetic polymorphisms on coronary artery disease susceptibility in Taiwanese subjects. Int. J. Med. Sci. 12, 510-516, https://doi.org/10.7150/ijms.12097 (2015).

21. Yin, D. L. et al. Association between the ICAM-1 gene polymorphism and coronary heart disease risk: a metaanalysis. Biosci. Rep. 39, BSR20180923, https://doi.org/10.1042/BSR20180923 (2019).

22. Yang, M. et al. Association between the polymorphisms in intercellular adhesion molecule-1 and the risk of coronary atherosclerosis: a case-controlled study. PLoS One 9, e109658, https://doi.org/10.1371/journal.pone.0109658 (2014).

23. Thayer, J. F., Yamamoto, S. S. \& Brosschot, J. F. The relationship of autonomic imbalance, heart rate variability and cardiovascular disease risk factors. Int. J. Cardiol. 141, 122-131, https://doi.org/10.1016/j.ijcard.2009.09.543 (2010). 
24. Nanchen, D. et al. Resting heart rate and the risk of heart failure in healthy adults: the Rotterdam Study. Circ. Heart Fail. 6, 403-410, https://doi.org/10.1161/CIRCHEARTFAILURE.112.000171 (2013).

25. Christofaro, D. G. D., Casonatto, J., Vanderlei, L. C. M, Cucato, G. G. \& Dias, R. M. R. Relationship between resting heart rate, blood pressure and pulse pressure in adolescents. Arq. Bras. Cardiol. 108, 405-410, https://doi.org/10.5935/abc.20170050 (2017).

26. Böhm, M., Reil, J. C., Deedwania, P., Kim, J. B. \& Borer, J. S. Resting heart rate: risk indicator and emerging risk factor in cardiovascular disease. Am. J. Med. 128, 219-228, https://doi.org/10.1016/j.amjmed.2014.09.016 (2015).

27. Custodis, F. et al. Vascular pathophysiology in response to increased heart rate. J. Am. Coll. Cardiol. 56, 19731983, https://doi.org/10.1016/j.jacc.2010.09.014 (2010).

28. Fox, K. et al. Resting heart rate in cardiovascular disease. J. Am. Coll. Cardiol. 50, 823-830, https://doi.org/10.1016/j.jacc.2007.04.079 (2007).

29. Heusch, G. Heart rate in the pathophysiology of coronary blood flow and myocardial ischaemia: benefit from selective bradycardic agents. Br. J. Pharmacol. 153, 1589-1601, https://doi.org/10.1038/sj.bjp.0707673 (2008).

30. Palatini, P. Heart rate as an independent risk factor for cardiovascular disease: current evidence and basic mechanisms. Drugs 67, 3-13, https://doi.org/10.2165/00003495-200767002-00002 (2007).

31. Reil, J. C. \& Böhm, M. The role of heart rate in the development of cardiovascular disease. Clin Res Cardiol 96, 585-592, https://doi.org/10.1007/s00392-007-0537-5 (2007).

32. Evans, K. L. et al. Genetics of heart rate in heart failure patients (GenHRate). Hum. Genomics 13, 22. https://doi.org/10.1186/s40246-019-0206-6 (2019).

33. Nasibullin, T. R. et al. Genotype/allelic combinations as potential predictors of myocardial infarction. Mol. Biol. Rep. 43, 11-16, https://doi.org/10.1007/s11033-015-3933-3 (2016).

34. Nepal, G., Yadav, J. K. \& Kong, Y. Association between K469E polymorphism of ICAM-1 gene and susceptibility of ischemic stroke: an updated meta-analysis. Mol. Genet. Genomic Med. 7, e00784, https://doi.org/10.1002/mgg3.784 (2019).

35. Expert panel on detection, evaluation, and treatment of high blood cholesterol in adults. Executive summary of the third report of the National Cholesterol Education Program (NCEP) expert panel on detection, evaluation, and treatment of high blood cholesterol in adults (Adult Treatment Panel III). JAMA 285, 2486-2497, https://doi.org/ 10.1001/jama.285.19.2486 (2001).

36. World Health Organization. Regional Office for the Western, Pacific. The Asia-Pacific perspective: redefining obesity and its treatment. https://apps.who.int/iris/handle/10665/206936/ (2000). 
37. World Health Organization. Appropriate body-mass index for Asian populations and its implications for policy and intervention strategies. Lancet 363, 157-163, https://doi.org/10.1016/S0140-6736(03)15268-3 (2004).

38. Friedewald, W. T., Levy, R. I. \& Fredrickson, D. S. Estimation of the concentration of low-density lipoprotein cholesterol in plasma, without use of the preparative ultracentrifuge. Clin. Chem. 18, 499-502, http://clinchem.aaccjnls.org/content/18/6/499.abstract (1972).

39. Sarecka-Hujar, B., Zak, I. \& Krauze, J. Interactions between rs5498 polymorphism in the ICAM1 gene and traditional risk factors influence susceptibility to coronary artery disease. Clin. Exp. Med. 9, 117-124, https://doi.org/org/10.1007/s10238-008-0022-0 (2009).

40. Iwao, M., Morisaki, H. \& Morisaki, T. Single-nucleotide polymorphism g.1548G>A (E469K) in human ICAM-1 gene affects mRNA splicing pattern and TPA-induced apoptosis. Biochem. Biophys. Res. Commun. 317, $729-735$. https://doi.org/org/10.1016/j.bbrc.2004.03.101 (2004).

41. Klop, B., Elte, J. W. F. \& Castro Cabezas, M. Dyslipidemia in obesity: mechanisms and potential targets. Nutrients 5, 1218-1240, https://doi.org/10.3390/nu5041218 (2013).

42. Liu, L. Z., Wu, E. P. \& Liu, H. L. Relation between K469E gene polymorphism of ICAM-1 and recurrence of ACS and cardiovascular mortality. Asian Pac. J. Trop. Med. 6, 916-920, https://doi.org/10.1016/S19957645(13)60164-9 (2013).

43. Gaetani, E. et al. The K469E polymorphism of the ICAM-1 gene is a risk factor for peripheral arterial occlusive disease. Blood Coagul. Fibrinolysis 13, 483-488. https://doi.org/10.1097/00001721-200209000-00002 (2002).

44. Nanchen, D. et al. Resting heart rate and incident heart failure and cardiovascular mortality in older adults: role of inflammation and endothelial dysfunction: the PROSPER study. Eur. J. Heart Fail. 15, 581-588, https://doi.org/10.1093/eurjhf/hfs195 (2013).

45. Al-Khatib, S. M. et al. 2017 AHA/ACC/HRS guideline for management of patients with ventricular arrhythmias and the prevention of sudden cardiac death: a report of the American College of Cardiology/American Heart Association task force on clinical practice guidelines and the Heart Rhythm Society. Circulation 138, e272-e391, https://doi.org/10.1161/CIR.0000000000000549 (2018).

46. Page, R. L. et al. 2015 ACC/AHA/HRS guideline for the management of adult patients with supraventricular tachycardia: a report of the American College of Cardiology/American Heart Association task force on clinical practice guidelines and the Heart Rhythm Society. Circulation 133, e506-574, https://doi.org/10.1161/CIR.0000000000000311 (2016). 
47. Schroeder, E. B. et al. Hypertension, blood pressure, and heart rate variability: the Atherosclerosis Risk in Communities (ARIC) study. Hypertension 42, 1106-1111, https://doi.org/10.1161/01.HYP.0000100444.71069.73 (2003).

48. Karason, K., Mølgaard, H., Wikstrand, J. \& Sjöström, L. Heart rate variability in obesity and the effect of weight loss. Am. J. Cardiol. 83, 1242-1247, https://doi.org/10.1016/S0002-9149(99)00066-1 (1999).

49. Kupari, M., Virolainen, J., Koskinen, P. \& Tikkanen, M. J. Short-term heart rate variability and factors modifying the risk of coronary artery disease in a population sample. Am. J. Cardiol. 72, 897-903, https://doi.org/10.1016/S0002-9149(99)00066-1 (1993).

50. Christensen, J. H., Toft, E., Christensen, M. S. \& Schmidt, E. B. Heart rate variability and plasma lipids in men with and without ischaemic heart disease. Atherosclerosis 145, 181-186, https://doi.org/10.1016/S00219150(99)00052-0 (1999).

\section{Acknowledgements}

This work was supported by the Faculty of Tropical Medicine and the Young Researcher Development Program 2019 of the National Research Council of Thailand (NRCT). We appreciatively acknowledge the nurses at Nong Waeng Health Promoting Hospital for assisting in collecting demographic data and blood samples. We also thank the collaboration of all participants in Sung Noen District, Nakhon Ratchasima Province, Thailand, We are grateful to the Faculty of Tropical Medicine for providing the equipment and facilities. We thank Mark Cleasby, PhD, from Edanz Group (www.edanzediting.com/ac) for editing a draft of this manuscript.

\section{Author contributions}

N.W. conceived and designed the research; N.W., P.P.P. and J.L. conducted data collection; N.W. performed all analyses and experiment; A.A., P.P., N.S. and K.K. supervised the research project; N.W. drafted the manuscript; A.A., P.P., N.S. and K.K. provided substantive review and edits to the manuscript; all authors approved the final version of this manuscript.

\section{Competing interests}

The authors declare no competing interests. 
Figure 1. DNA sequencing chromatograph for the ICAM1 (a) rs5498 (A>G) polymorphism and (b) rs281432 (C>G) polymorphism. The ICAM1 rs5498 (a1) wild-type homozygous genotype: AA; (a2) heterozygous genotype: AG; (a3) homozygous genotype: GG. The ICAM1 rs281432 (b1) wild-type homozygous genotype: CC; (b2) heterozygous genotype: CG); (b3) homozygous genotype: GG.

Figure 2. Relationships between the ICAM1 rs5498 polymorphism genotype in the dominant model and ASCVD risk factors. Normal control (wild-type AA, $n=91$; variant AG+GG, $n=52$ ) and HCL (wild-type AA, $n=63$; variant AG+GG, $\mathrm{n}=72$ ) groups. (A) Heart rate. ${ }^{\#} \mathrm{P}<0.001$ vs. normal control (AA). (B) BMI $(\mathrm{P}=0.042$ for trend). (C) TC (D) LDL-C. ${ }^{\#} \mathrm{P}<0.001$ vs. normal control (AA), ${ }^{\S} \mathrm{P}<0.001$ vs. normal control (AG+GG). (E) Triglycerides. ${ }^{\#} \mathrm{P}<0.001$ vs. normal control (AA), ${ }^{\S} \mathrm{P}<0.001 v s$. normal control (AG+GG), ${ }^{\ddagger} \mathrm{P}<0.001$ vs. HCL (AA). (F) TG/HDL-C ratio. ${ }^{\#} \mathrm{P}<$ $0.001 v s$. normal control (AA), ${ }^{\S} \mathrm{P}<0.001$ vs. normal control (AG+GG). (G) HDL-C. NS, no significance. (H) ICAM1 mRNA expression. ${ }^{\#} \mathrm{P}<0.05$ vs. normal control (AA), ${ }^{\S} \mathrm{P}<0.05$ vs. HCL (AA).

Figure 3. The relationships among ASCVD risk factors, oxidative stress, endothelial dysfunction, autonomic imbalance and genetic variation 
Table 1 Clinical and laboratory characteristics of the participants with HCL and normal controls

\begin{tabular}{|c|c|c|c|}
\hline Variable & Normal control & HCL & $\mathrm{P}$ value \\
\hline $\mathrm{N}$ & 143 & 135 & - \\
\hline Age, (years) & $46.56 \pm 6.47$ & $48.06 \pm 6.00$ & 0.065 \\
\hline Male $(\%)$ & 32.9 & 28.1 & 0.393 \\
\hline Smoker $(\%)$ & 21.7 & 25.9 & 0.405 \\
\hline Current alcohol consumer $(\%)$ & 37.8 & 40.7 & 0.611 \\
\hline Exercise $(\%)$ & 9.1 & 8.9 & 0.953 \\
\hline $\mathrm{SBP}(\mathrm{mmHg})$ & $122.42 \pm 14.70$ & $128.48 \pm 15.30$ & 0.001 \\
\hline $\mathrm{DBP}(\mathrm{mmHg})$ & $76.59 \pm 10.05$ & $80.13 \pm 9.87$ & 0.003 \\
\hline RHR (beats/min) & $77.44 \pm 12.13$ & $81.04 \pm 11.53$ & 0.012 \\
\hline $\operatorname{BMI}\left(\mathrm{kg} / \mathrm{m}^{2}\right)$ & $25.70 \pm 5.01$ & $27.30 \pm 4.62$ & 0.006 \\
\hline $\mathrm{WC}(\mathrm{cm})$ & $85.70 \pm 12.31$ & $89.27 \pm 10.34$ & 0.009 \\
\hline $\mathrm{HC}(\mathrm{cm})$ & $95.59 \pm 10.31$ & $97.87 \pm 8.89$ & 0.049 \\
\hline WHR & $0.89 \pm 0.06$ & $0.91 \pm 0.06$ & 0.023 \\
\hline $\mathrm{TC}(\mathrm{mmol} / \mathrm{L})$ & $4.30 \pm 0.65$ & $5.47 \pm 1.01$ & 0.000 \\
\hline LDL-C (mmol/L) & $2.48 \pm 0.53$ & $3.33 \pm 0.90$ & 0.000 \\
\hline HDL-C (mmol/L) & $1.30 \pm 0.32$ & $1.25 \pm 0.34$ & 0.218 \\
\hline $\mathrm{TG}(\mathrm{mmol} / \mathrm{L})$ & $1.10(0.41,2.94)$ & $1.90(0.60,4.46)$ & $0.000 *$ \\
\hline TG/HDL-C (mmol/L) & $0.89(0.22,2.44)$ & $1.49(0.34,8.62)$ & $0.000 *$ \\
\hline ICAM1 mRNA expression (arbitrary units) & $1.15(0.14,9.19)$ & $1.51(0.15,9.97)$ & $0.096 *$ \\
\hline
\end{tabular}

$\mathrm{P}$ values calculated using the $\chi^{2}$ test (categorical variables) or ANOVA (continuous variables with normal distribution). *P values calculated using the non-parametric median scores test (continuous variables with non-normal distribution). A smoker was defined who had smoked at least 100 cigarettes in their lifetime. Moderate and heavy alcohol drinking were classified as a current alcohol consumer. Exercise was defined as activity of a moderate or vigorous intensity for $\geq 30 \mathrm{~min} /$ day on at least 3 days a week. 
Table 2 Genetic models analysis for the ICAM1 rs5498 and rs281432 polymorphisms in HCL and normal participants

\begin{tabular}{|c|c|c|c|c|c|}
\hline Variable & $\begin{array}{l}\text { All } \\
\text { participants } \\
(\mathrm{n}=278)\end{array}$ & $\begin{array}{l}\text { Normal } \\
\text { control } \\
(\mathrm{n}=143)\end{array}$ & $\begin{array}{l}\text { HCL } \\
(n=135)\end{array}$ & OR $(95 \% \mathrm{CI})$ & $\mathrm{P}$ value \\
\hline \multicolumn{6}{|c|}{ ICAM1 rs5498 allele, $\mathrm{n}(\%)$} \\
\hline A & $408(73.0)$ & $224(78.0)$ & $184(68.0)$ & (Reference) & \\
\hline G & $148(27.0)$ & $62(22.0)$ & $86(32.0)$ & $1.69(1.15-2.47)$ & 0.007 \\
\hline$P_{\mathrm{HWE}}$ & 0.220 & 1.000 & 0.140 & & \\
\hline
\end{tabular}

ICAM1 rs5498 genotypes

Additive effects, $\mathrm{n}(\%)$

$\begin{array}{llllll}\text { AA } & 154(55.0) & 91(64.0) & 63(47.0) & \text { (Reference) } & \\ \text { AG } & 100(36.0) & 42(29.0) & 58(43.0) & 1.99(1.20-3.32) & 0.008 \\ \text { GG } & 24(9.0) & 10(7.0) & 14(10.0) & 2.02(0.84-4.84) & 0.114\end{array}$

Dominant effects, n (\%)

$\begin{array}{llllll}\text { AA } & 154(55.0) & 91(64.0) & 63(47.0) & \text { (Reference) } & \\ \text { AG+GG } & 124(45.0) & 52(36.0) & 72(53.0) & 2.00(1.24-3.23) & 0.005\end{array}$

Recessive effects, n (\%)

$\begin{array}{llllll}\mathrm{AA}+\mathrm{AG} & 254(91.0) & 133(93.0) & 121(90.0) & \text { (Reference) } & \\ \mathrm{GG} & 24(9.0) & 10(7.0) & 14(10.0) & 1.54(0.66-3.59) & 0.319\end{array}$

ICAM1 rs281432 allele, n (\%)

$\begin{array}{rllllll} & \mathrm{C} & 362(65.0) & 185(65.0) & 177(66.0) & \text { (Reference) } & \\ & \mathrm{G} & 194(35.0) & 101(35.0) & 93(34.0) & 0.96(0.68-1.36) & 0.859 \\ P_{\text {HWE }} & & 0.360 & 0.580 & 0.570 & & \end{array}$

ICAM1 rs281432 genotypes

Additive effects, n (\%)

$\begin{array}{llllll}\text { CC } & 114(41.0) & 58(41.0) & 56(41.0) & (\text { Reference }) & \\ \text { CG } & 134(48.2) & 69(48.0) & 65(48.0) & 0.98(0.59-1.61) & 0.923 \\ \text { GG } & 30(10.8) & 16(11.0) & 14(10.0) & 0.91(0.40-2.03) & 0.811\end{array}$

Dominant effects, n (\%)

$\begin{array}{llllll}\mathrm{CC} & 114(41.0) & 58(41.0) & 56(41.0) & \text { (Reference) } & \\ \mathrm{CG}+\mathrm{GG} & 164(59.0) & 85(59.0) & 79(58.0) & 0.96(0.60-1.55) & 0.876\end{array}$

Recessive effects, n (\%)

$\begin{array}{llllll}\mathrm{CC}+\mathrm{CG} & 248(89.2) & 127(89.0) & 121(89.0) & \text { (Reference) } & \\ \mathrm{GG} & 30(10.8) & 16(11.0) & 14(10.0) & 0.92(0.43-1.96) & 0.826\end{array}$

$\mathrm{P}$ values assessed using odds ratios, according to the genetic model. $\mathrm{P}_{\mathrm{HWE}}$ for the Hardy-Weinberg equilibrium test. Genetic models: additive model (wild-type $v s$. heterozygous variants and wild-type $v s$. homozygous variants), dominant model (wild type $v s$. heterozygous variants + homozygous variants), and recessive model (wild-type + heterozygous variants vs. homozygous variants). 
Table 3 Clinical and laboratory characteristics of participants with rs5498 or rs281432 ICAM1 variants in the dominant model

\begin{tabular}{|c|c|c|c|c|c|c|}
\hline \multirow{2}{*}{ Variable } & \multicolumn{3}{|l|}{ ICAM1 rs5498 } & \multicolumn{3}{|l|}{ ICAM1 rs281432 } \\
\hline & AA $(\mathrm{n}=154)$ & $\mathrm{AG}+\mathrm{GG}(\mathrm{n}=124)$ & $P$ value & $\mathrm{CC}(\mathrm{n}=114)$ & CG + GG $(n=164)$ & $P$ value \\
\hline Age (years) & $47.66 \pm 6.40$ & $46.81 \pm 6.13$ & 0.264 & $47.65 \pm 5.92$ & $47.04 \pm 6.53$ & 0.428 \\
\hline Male (\%) & 34.4 & 25.8 & 0.121 & 28.1 & 32.3 & 0.450 \\
\hline Smoker $(\%)$ & 28.6 & 17.7 & 0.035 & 24.6 & 23.2 & 0.789 \\
\hline $\begin{array}{l}\text { Current alcohol consumer } \\
(\%)\end{array}$ & 40.3 & 37.9 & 0.689 & 41.2 & 37.8 & 0.565 \\
\hline Exercise (\%) & 9.7 & 8.1 & 0.627 & 9.6 & 8.5 & 0.750 \\
\hline $\mathrm{SBP}(\mathrm{mmHg})$ & $125.66 \pm 15.73$ & $124.99 \pm 14.73$ & 0.717 & $127.25 \pm 17.34$ & $124.05 \pm 13.55$ & 0.085 \\
\hline $\mathrm{DBP}(\mathrm{mmHg})$ & $78.38 \pm 9.71$ & $78.22 \pm 10.61$ & 0.892 & $79.17 \pm 11.29$ & $77.71 \pm 9.18$ & 0.257 \\
\hline RHR (beats/min) & $77.26 \pm 11.44$ & $81.58 \pm 12.19$ & 0.003 & $79.19 \pm 12.18$ & $79.18 \pm 11.83$ & 0.995 \\
\hline BMI $\left(\mathrm{kg} / \mathrm{m}^{2}\right)$ & $26.17 \pm 4.88$ & $26.87 \pm 4.87$ & 0.235 & $26.21 \pm 5.16$ & $26.66 \pm 4.69$ & 0.448 \\
\hline $\mathrm{WC}(\mathrm{cm})$ & $86.75 \pm 11.78$ & $88.28 \pm 11.16$ & 0.272 & $87.19 \pm 11.96$ & $87.60 \pm 11.23$ & 0.773 \\
\hline $\mathrm{HC}(\mathrm{cm})$ & $95.94 \pm 9.83$ & $97.65 \pm 9.48$ & 0.143 & $96.19 \pm 10.21$ & $97.05 \pm 9.33$ & 0.466 \\
\hline WHR & $0.90 \pm 0.06$ & $0.90 \pm 0.06$ & 0.972 & $0.91 \pm 0.06$ & $0.90 \pm 0.06$ & 0.607 \\
\hline $\mathrm{TC}(\mathrm{mmol} / \mathrm{L})$ & $4.70 \pm 1.00$ & $5.06 \pm 1.03$ & 0.004 & $4.88 \pm 1.13$ & $4.85 \pm 0.95$ & 0.812 \\
\hline LDL-C (mmol/L) & $2.75 \pm 0.81$ & $3.06 \pm 0.86$ & 0.002 & $2.87 \pm 0.89$ & $2.90 \pm 0.82$ & 0.724 \\
\hline HDL-C (mmol/L) & $1.27 \pm 0.34$ & $1.28 \pm 0.33$ & 0.824 & $1.28 \pm 0.35$ & $1.26 \pm 0.32$ & 0.609 \\
\hline $\mathrm{TG}(\mathrm{mmol} / \mathrm{L})$ & $1.33(0.41,4.46)$ & $1.33(0.47,3.99)$ & $0.740 *$ & $1.40(0.41,4.46)$ & $1.29(0.44,3.53)$ & $0.608^{*}$ \\
\hline TG/HDL-C ratio & $1.03(0.29,8.62)$ & $1.07(0.22,5.31)$ & $0.939^{*}$ & $1.07(0.30,8.62)$ & $1.05(0.22,6.90)$ & $0.917 *$ \\
\hline ICAM1 mRNA expression & $1.05(0.15,9.97)$ & $1.84(0.14,9.94)$ & $0.000^{*}$ & $1.14(0.14,9.97)$ & $1.46(0,9.92)$ & $0.302 *$ \\
\hline
\end{tabular}

$\mathrm{P}$ values calculated using Student's $t$ test (continuous variables with normal distribution); *P values calculated using the non-parametric median scores test (continuous variables with non-normal distribution). Dominant model analysis: wildtype (AA) vs. heterozygous + homozygous variants (AG+GG). 
Table 4 The effect of the interaction of the ICAMI rs5498 polymorphism and ASCVD risk factors

\begin{tabular}{|c|c|c|c|c|c|c|}
\hline \multirow{2}{*}{ Variable } & \multicolumn{2}{|c|}{ ASCVD risk factors } & \multirow[b]{2}{*}{$\mathrm{OR}^{\mathrm{a}}(95 \% \mathrm{CI})$} & \multirow[b]{2}{*}{$\mathrm{P}^{a}$ value } & \multirow[b]{2}{*}{$\mathrm{OR}^{\mathrm{b}}(95 \% \mathrm{CI})$} & \multirow[b]{2}{*}{$\mathrm{P}^{b}$ value } \\
\hline & Low-risk & High-risk & & & & \\
\hline \multicolumn{7}{|l|}{$75^{\text {th }}$ percentile RHR (beats/min), } \\
\hline $\mathrm{n}(\%)$ & $<86(\mathrm{n}=203)$ & $\geq 86(\mathrm{n}=75)$ & & & & \\
\hline \multicolumn{7}{|l|}{ ICAM1 rs5498 (A > G) } \\
\hline AA & $120(59.1)$ & $34(45.3)$ & (Reference) & & (Reference) & \\
\hline $\mathrm{AG}+\mathrm{GG}$ & $83(40.9)$ & $41(54.7)$ & $1.74(1.02-2.97)$ & 0.041 & $1.60(0.92-2.76)$ & 0.094 \\
\hline $\begin{array}{l}\text { Tachycardia at RHR (beats/min } \\
\text { n (\%) }\end{array}$ & \multicolumn{5}{|c|}{ Tachycardia at RHR (beats/min), } & \\
\hline \multicolumn{7}{|l|}{ ICAM1 rs5498 (A > G) } \\
\hline AA & $151(57.4)$ & $3(20.0)$ & (Reference) & & (Reference) & \\
\hline $\mathrm{AG}+\mathrm{GG}$ & $112(42.6)$ & $12(80.0)$ & $5.40(1.49-19.56)$ & 0.010 & $4.92(1.33-18.23)$ & 0.017 \\
\hline $\mathrm{TC}(\mathrm{mmol} / \mathrm{L}), \mathrm{n}(\%)$ & $<5.17(\mathrm{n}=175)$ & $\geq 5.17(\mathrm{n}=103)$ & & & & \\
\hline \multicolumn{7}{|l|}{ ICAM1 rs5498 (A > G) } \\
\hline AA & $103(58.9)$ & $51(49.5)$ & (Reference) & & (Reference) & \\
\hline $\mathrm{AG}+\mathrm{GG}$ & $72(41.1)$ & $52(50.5)$ & $1.59(0.96-2.63)$ & 0.071 & $1.62(0.97-2.71)$ & 0.067 \\
\hline LDL-C (mmol/L), n (\%) & $<3.36(\mathrm{n}=195)$ & $\geq 3.36(\mathrm{n}=80)$ & & & & \\
\hline \multicolumn{7}{|l|}{ ICAM1 rs5498 $(\mathrm{A}>\mathrm{G})$} \\
\hline AA & $119(61.0)$ & $32(40.0)$ & (Reference) & & (Reference) & \\
\hline \multirow[t]{3}{*}{$\mathrm{AG}+\mathrm{GG}$} & $76(39.0)$ & $48(60.0)$ & $1.99(1.15-3.44)$ & 0.014 & $2.08(1.19-3.65)$ & 0.011 \\
\hline & $>1.29$ (women) & $\leq 1.29$ (women) & & & & \\
\hline & $>1.04($ men $)$ & $\leq 1.04($ men $)$ & & & & \\
\hline HDL-C (mmol/L), n (\%) & $(n=149)$ & $(\mathrm{n}=129)$ & & & & \\
\hline \multicolumn{7}{|l|}{ ICAM1 rs5498 (A > G) } \\
\hline AA & $83(55.7)$ & $71(55.0)$ & (Reference) & & (Reference) & \\
\hline $\mathrm{AG}+\mathrm{GG}$ & $66(44.3)$ & $58(45.0)$ & $1.03(0.64-1.65)$ & 0.911 & $1.06(0.65-1.73)$ & 0.823 \\
\hline TG (mmol/L), n (\%) & $<1.69(\mathrm{n}=191)$ & $\geq 1.69(\mathrm{n}=87)$ & & & & \\
\hline \multicolumn{7}{|l|}{ ICAM1 rs5498 $(\mathrm{A}>\mathrm{G})$} \\
\hline AA & $106(55.5)$ & $48(55.2)$ & (Reference) & & (Reference) & \\
\hline $\mathrm{AG}+\mathrm{GG}$ & $85(44.5)$ & $39(44.8)$ & $1.01(0.61-1.69)$ & 0.960 & $1.08(0.62-1.87)$ & 0.786 \\
\hline TG/HDL-C (mmol/L), n (\%) & $<1.75(\mathrm{n}=214)$ & $\geq 1.75(\mathrm{n}=64)$ & & & & \\
\hline \multicolumn{7}{|l|}{ ICAM1 rs5498 $(\mathrm{A}>\mathrm{G})$} \\
\hline AA & $119(55.6)$ & $35(54.7)$ & (Reference) & & (Reference) & \\
\hline $\mathrm{AG}+\mathrm{GG}$ & $95(44.4)$ & $29(45.3)$ & $1.04(0.59-1.82)$ & 0.897 & $1.16(0.64-2.10)$ & 0.624 \\
\hline \multicolumn{7}{|l|}{$75^{\text {th }}$ percentile (arbitrary units) } \\
\hline ICAM1 mRNA expression, (n) & $<3.12(\mathrm{n}=206)$ & $\geq 3.12(\mathrm{n}=72)$ & & & & \\
\hline \multicolumn{7}{|l|}{ ICAM1 rs5498 $(\mathrm{A}>\mathrm{G})$} \\
\hline AA & $127(61.7)$ & $27(37.5)$ & (Reference) & & (Reference) & \\
\hline $\mathrm{AG}+\mathrm{GG}$ & $79(38.3)$ & $45(62.5)$ & $2.68(1.54-4.66)$ & 0.000 & $2.47(1.41-4.33)$ & 0.002 \\
\hline
\end{tabular}

$\mathrm{OR}^{\mathrm{a}}$ Unadjusted. $\mathrm{OR}^{\mathrm{b}}$ Adjusted for sex, age, smoking status, alcohol consumption status, and exercise. $\mathrm{P}^{a}$ value associated with odds ratio $(\mathrm{OR})^{\mathrm{a}}$. $\mathrm{P}^{b}$ value associated with $\mathrm{OR}^{\mathrm{b}}$. 


\section{Figures}

A

(A1)

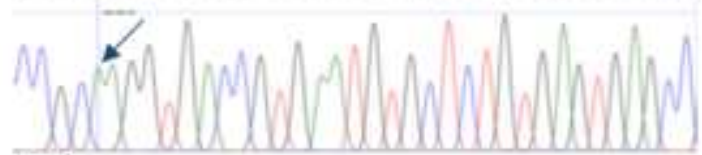

(A2)

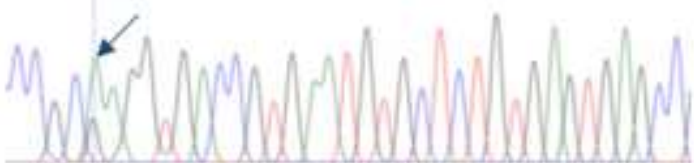

(A3)

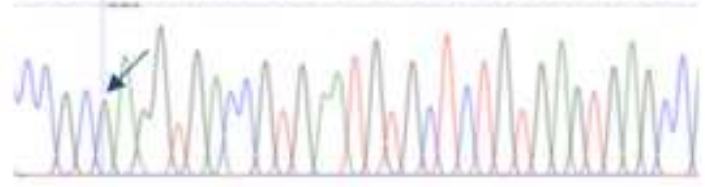

B

(B1)

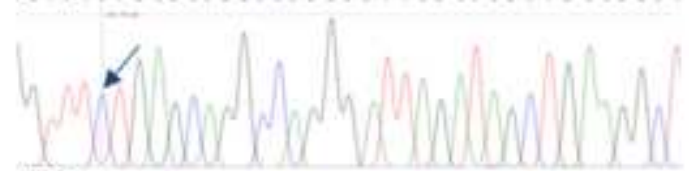

(B2)

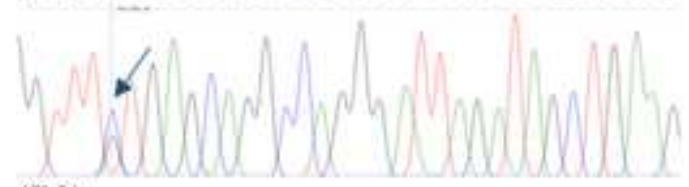

(B3)

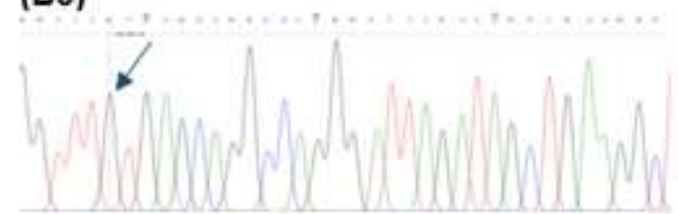

\section{Figure 1}

DNA sequencing chromatograph for the ICAM1 (a) rs5498 (A>G) polymorphism and (b) rs281432 (C>G) polymorphism. The ICAM1 rs5498 (a1) wild-type homozygous genotype: AA; (a2) heterozygous genotype: AG; (a3) homozygous genotype: GG. The ICAM1 rs281432 (b1) wild-type homozygous genotype: CC; (b2) heterozygous genotype: CG); (b3) homozygous genotype: GG. 


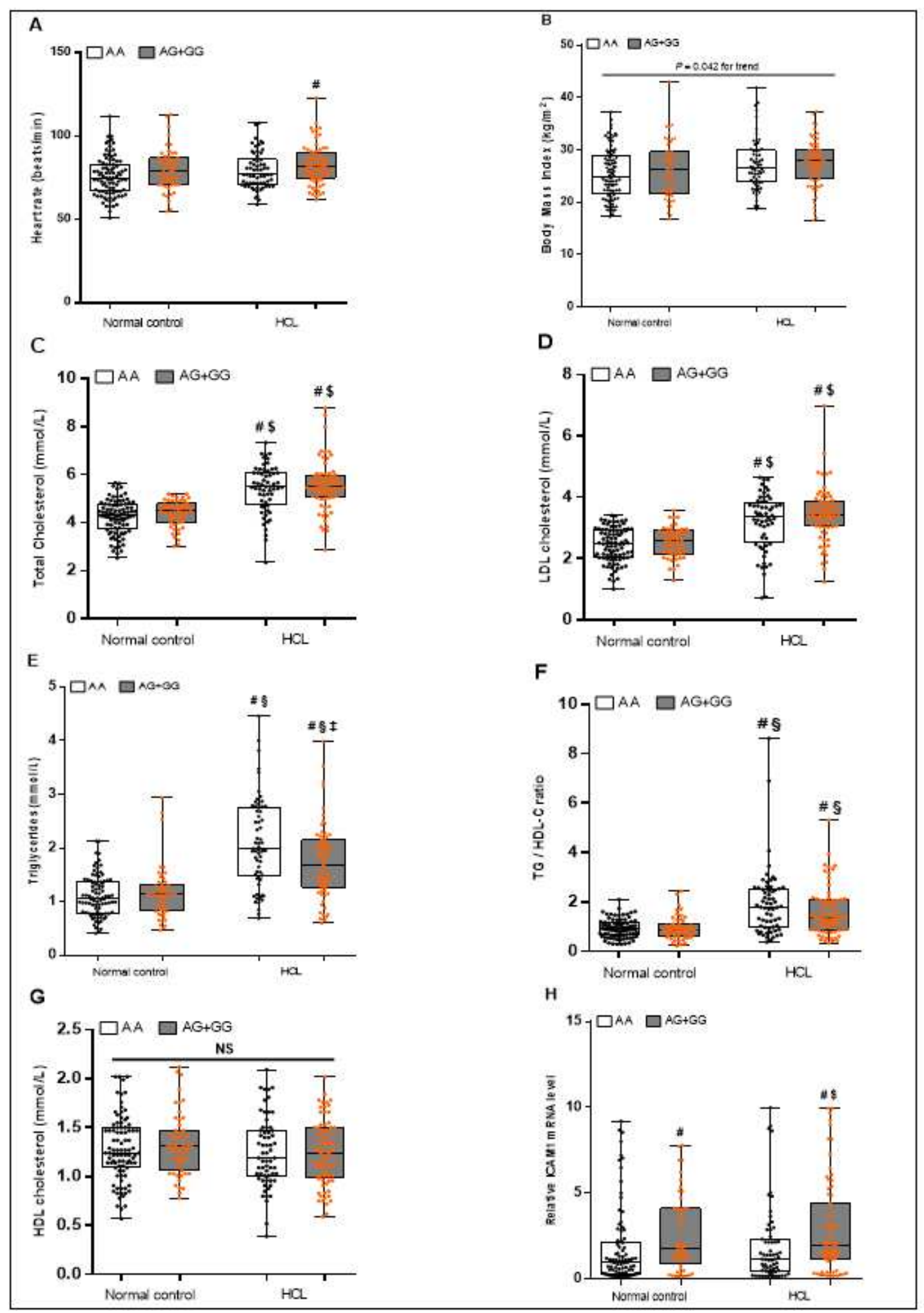

Figure 2

Relationships between the ICAM1 rs5498 polymorphism genotype in the dominant model and ASCVD risk factors Normal control (wild-type $A A, n=91$; variant $A G+G G, n=52$ ) and $H C L$ (wild-type $A A, n=63$; variant $A G+G G, n=72$ ) groups. (A) Heart rate. $\# P<0.001$ vs. normal control (AA). (B) $B M I(P=0.042$ for trend). (C) TC (D) LDL-C. \#P < 0.001 vs. normal control (AA), §P < 0.001 vs. normal control ( $A G+G G)$. (E) Triglycerides. \#P $<0.001$ vs. normal control (AA), §P $<0.001$ vs. normal control (AG+GG), $¥ P<0.001$ vs. 
HCL (AA). (F) TG/HDL-C ratio. \#P < 0.001 vs. normal control (AA), §P < 0.001 vs. normal control (AG+GG). (G) HDL-C. NS, no significance. (H) ICAM1 mRNA expression. \#P $<0.05$ vs. normal control (AA), §P $<0.05$ vs. HCL (AA).

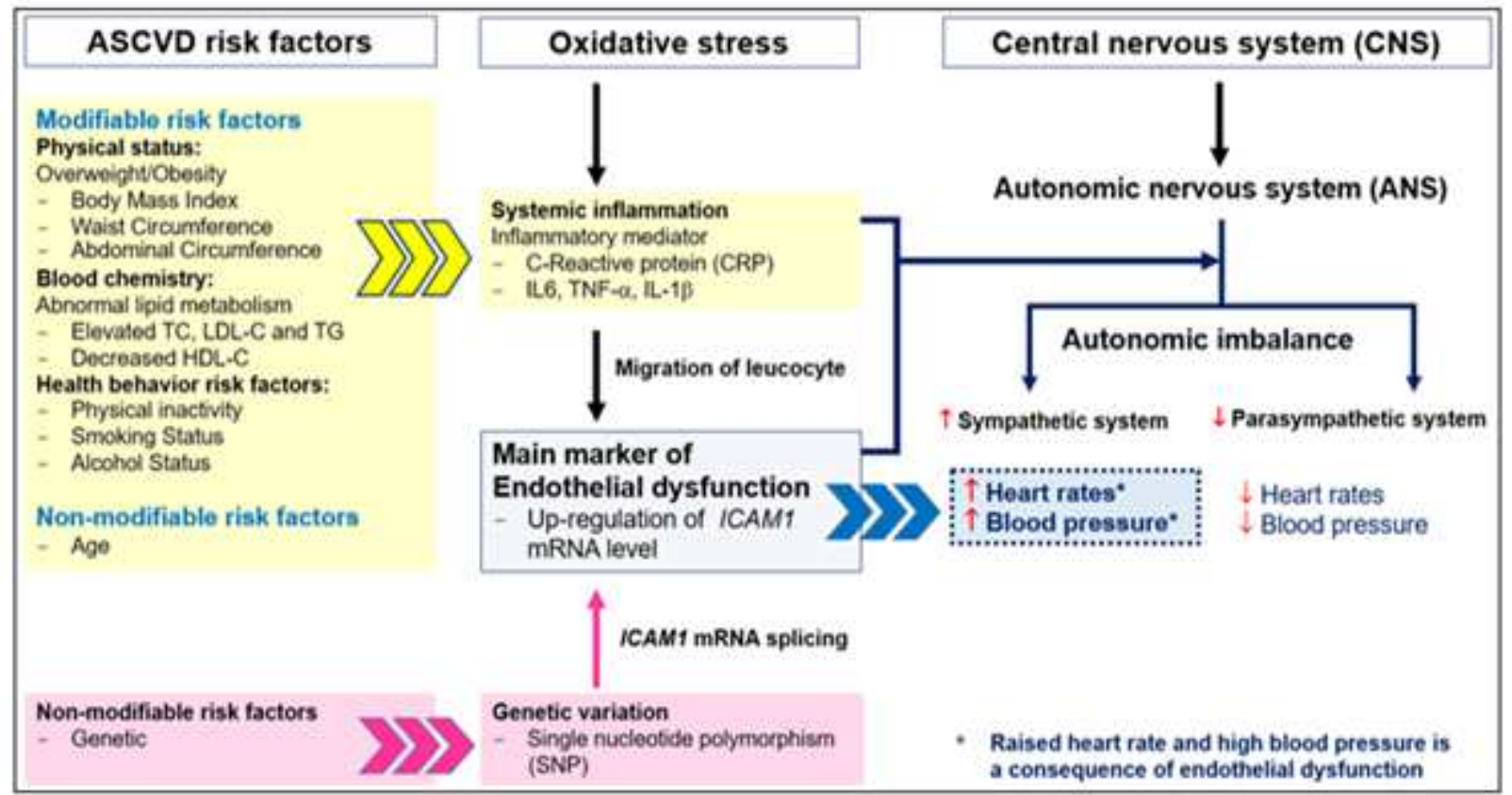

\section{Figure 3}

The relationships among ASCVD risk factors, oxidative stress, endothelial dysfunction, autonomic imbalance and genetic variation. 